\title{
Choques estocásticos na renda mundial e os efeitos na economia brasileira
}

Celso José Costa Junior*

Resumo: Este trabalho tem o objetivo principal de estudar o comportamento do produto brasileiro em relação a um choque na renda mundial. Para essa finalidade, usou-se a abordagem DSGE. Os parâmetros foram obtidos usando o método de calibragem e a análise dos resultados foi feita através de funções impulso-resposta. A característica básica deste artigo é que o choque na renda mundial expande as exportações líquidas, as quais se tornam o canal de retorno das outras variáveis do modelo ao estado estacionário. Os resultados obtidos demonstram que não ocorreram grandes alterações no produto, apenas um rearranjamento entre os fatores de produção (trabalho e capital), contudo essas duas variáveis retornam ao estado estacionário ainda no período de simulação.

Palavras-chave: Modelos DSGE; Choques na Renda Mundial; Exportações Líquidas Classificação JEL: F41, C63, E37.

\footnotetext{
* Doutorando em Desenvolvimento Econômico da UFPR e Professor da Escola de Negócios da Universidade Positivo.
} 


\section{Introdução}

Todas as economias estão sujeitas a choques externos. Entretanto, a presença de choques externos grandes e frequentes torna essa questão particularmente relevante para os países em desenvolvimento. Nesses países, a instabilidade oriunda de fatores externos torna ainda mais árdua à tarefa de promover o crescimento.

Percebendo que existe a necessidade de estudos que analisam o impacto nas principais variáveis macroeconômicas, devido a choques externos, este trabalho tem o objetivo de estudar o comportamento da economia brasileira em relação a um choque no nível de renda mundial, o qual aumentaria as exportações líquidas brasileiras. Um estudo sobre esse tema justifica-se pela importância das exportações líquidas no crescimento econômico dos países. Nota-se que nos momentos em que o crescimento da economia brasileira esteve superior ao crescimento da economia americana (figura 1) as exportações líquidas apresentaram um crescimento negativo, isso devido às importações crescerem mais do que as exportações.

Figura 1- Gráficos dos Crescimentos do NX, do PIB e do PIB dos EUA.
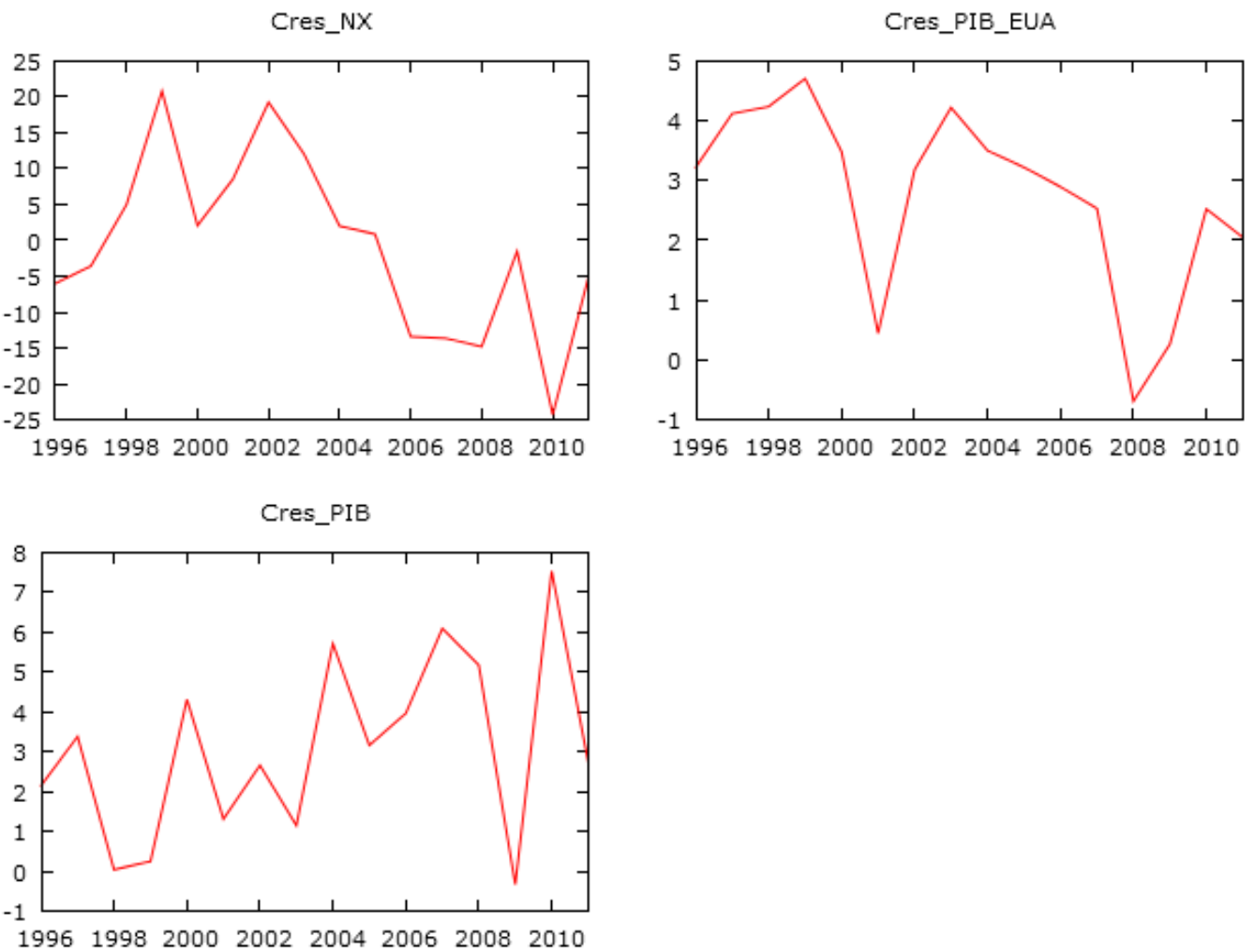

Fonte: Ipeadata (2013)

Ao longo dos últimos vinte anos houve um enorme avanço nas ferramentas matemáticas, estatísticas, probabilísticas e computacionais disponíveis para os macroeconomistas aplicados. Este enorme conjunto de ferramentas mudou a 
forma como os pesquisadores abordam testes de modelos, validam teorias ou simplesmente buscam regularidades em dados. As expectativas racionais e as revoluções de calibração e de estimação também forçaram os pesquisadores a tentar construir uma ponte mais sólida entre trabalhos teóricos e aplicados, uma ponte que muitas vezes esteve ausente em grande parte dos exercícios aplicados realizados na década de 1970 e 1980 (Canova, 2007). O trabalho de Kydland e Prescott (1982) "Time to Build and Aggregate Fluctuations" revolucionou a macroeconomia moderna, contudo os primeiros passos dessa metodologia foram dados por Ramsey (1927, 1928), Cass (1965), Koopmans (1965) e Brock e Mirman (1972).

Geralmente, a abordagem da calibragem para obter os valores dos parâmetros não é a mais apropriada, pois seus valores obtidos estão sempre condicionados a um modelo particular. Então, não é indicado importar valores de um outro modelo. Devido a isso, a estimação de modelos DSGE por meio de metodologias Bayesianas tornou-se o método de estimação mais comum entre os macroeconomistas aplicados. Contudo, os parâmetros calibrados neste trabalho foram obtidos de um trabalho com a estrutura similar a este (Costa Junior et al, 2012), evitando os problemas apresentados acima.

Além dessa seção introdutória, este trabalho está estruturado da seguinte forma: a seção dois descreve o modelo DSGE; a terceira seção trata da calibração dos parâmetros do modelo; a seção quatro demonstra os resultados encontrados; e por fim, são apresentadas as conclusões.

\section{Modelo}

Nesta seção é apresentado o modelo econômico deste trabalho. Trata-se de um modelo simples formado por famílias, firmas e setor externo ${ }^{1}$.

\subsection{Famílias}

O primeiro agente desse modelo é o agente representativo famílias. Esse agente maximiza sua função utilidade (que representa sua felicidade instantânea) escolhendo consumo e lazer, sujeita a sua restrição orçamentária.

\subsubsection{Consumidores}

É suposto que cada agente maximiza sua utilidade intertemporal escolhendo consumo, $\left\{C_{t}\right\}_{t=0}^{\infty}$, e lazer, $\left\{1-L_{t}\right\}_{t=0}^{\infty}$. Assim, suas preferências são definidas pela seguinte

\footnotetext{
1 A ideia de deixar o modelo simples é manter a concentração nas principais variáveis deste trabalho, C e Y. Considerar outras formas de rigidez (mercado em concorrência imperfeita, hábitos de consumo etc) aumentaria o custo de resolução, porém os resultados não seriam substancialmente diferentes. Então, a escolha foi manter o modelo o mais simples possível.
} 
função utilidade:

$$
U=E_{t} \sum_{t=0}^{\infty} \beta\left[\gamma \log C_{t}+(1-\gamma) \log \left(1-L_{i, t}\right)\right]
$$

onde $E_{t}$ é o operador de expectativas, $\beta$ é a taxa de desconto intertemporal, $\gamma \in(0,1)$ é a participação do consumo na utilidade das famílias.

A restrição orçamentária diz que o consumo mais o investimento, $I_{t}$, não podem exceder a soma das receitas vindas do trabalho e do capital:

$$
\left(C_{t}+I_{t}\right) P_{t}=W_{t} L_{t}+R_{t} K_{t}
$$

onde $W_{t}$ é o salário, $R_{t}$ é a taxa de retorno do capital, $K_{t}$ é o estoque de capital, $L_{t}$ é a quantidade de trabalho e $P_{t}$ é o nível de preços, o qual é normalizado a um.

O processo de acumulação de capital é definido por:

$$
K_{t+1}=(1-\delta) K_{t}+I_{t}
$$

onde $\delta$ é a taxa de depreciação.

Usando (2) em (1), obtêm-se a restrição orçamentária das famílias:

$$
C_{t}+K_{t+1}=W_{t} L_{t}+\left(R_{t}+1-\delta\right) K_{t}
$$

O lagrangiano correspondente ao problema enfrentado pelos consumidores ricardianos é o seguinte:

$$
\underset{\left(C_{t}, L_{t}, K_{t}\right)}{M A X} \operatorname{Lagr}=E_{t} \sum_{t=0}^{\infty} \beta\left\{\begin{array}{c}
\gamma \log C_{t}+(1-\gamma) \log \left(1-L_{t}\right) \\
-\lambda_{t}\left[C_{t}+K_{t+1}-W_{t} L_{t}-\left(R_{t}+1-\delta\right) K_{t}\right]
\end{array}\right\}
$$

Assim, chega-se as condições de primeira ordem do problema acima:

$$
\begin{gathered}
\frac{\partial \operatorname{Lagr} r}{\partial C_{t}}=\frac{\gamma}{C_{t}}-\lambda_{t}=0 \\
\frac{\partial \operatorname{Lagr}}{\partial L_{t}}=-\frac{(1-\gamma)}{\left(1-L_{t}\right)}+\lambda_{t} W_{t}=0 \\
\frac{\partial \operatorname{Lagr} r}{\partial K_{t}}=E_{t+1} \beta \lambda_{t+1}\left[R_{t+1}+1-\delta\right]-\lambda_{t}=0
\end{gathered}
$$

Combinando as equações (4) e (5), obtem-se a equação da oferta de trabalho dos consumidores ricardianos:

$$
\frac{1-\gamma}{\gamma} \frac{C_{t}}{1-L_{t}}=W_{t}
$$

E usando as equações (4) e (6), chega-se a equação de Euler para o consumo:

$$
\frac{1}{C_{t}}=\beta E_{t+1} \frac{1}{C_{t+1}}\left(R_{t+1}+1-\delta\right)
$$




\subsection{Firmas}

O problema das firmas consiste em escolher valores ótimos de utilização dos fatores de produção capital e trabalho. Supõe-se que tanto os mercados de bens e serviços como os mercados de fatores são perfeitamente competitivos. As firmas adquirem capital e trabalho das famílias com o objetivo de maximizar seu lucro, tomando como dados os preços dos mesmos. A função de produção é dada por:

$$
Y_{t}=A_{t} K_{t}^{\alpha} L_{t}^{1-\alpha}
$$

onde $A_{t}$ é a produtividade total dos fatores, $\alpha$ é a participação do capital no produto, $K_{t}$ é o estoque de capital, $L_{t}$ é a quantidade de horas trabalhadas e $Y_{t}$ é o produto.

A produtividade ${ }^{2}$ segue um processo estocástico $\mathrm{AR}(1)$ descrito abaixo:

$$
A_{t}=\rho_{A} A_{t-1}+\varepsilon_{A, 1}
$$

onde $\varepsilon_{A, t}$ é o termo de erro.

O problema da firma é maximizar a sua função lucro:

$$
\pi_{t}=A_{t} K_{t}^{\alpha} L_{t}^{1-\alpha}-W_{t} L_{t}-R_{t} K_{t}
$$

Do problema de maximização acima são obtidas as seguintes condições de primeira ordem:

$$
\begin{array}{r}
\frac{\partial \pi_{t}}{\partial K_{t}}=\alpha A_{t} K_{t}^{\alpha-1} L_{t}^{1-\alpha}-R_{t}=0 \\
\frac{\partial \pi_{t}}{\partial L_{t}}=(1-\alpha) A_{t} K_{t}^{\alpha} L_{t}^{-\alpha}-W_{t}=0
\end{array}
$$

Das equações (12) e (13), resultam as equações dos preços dos fatores de produção:

$$
\begin{array}{r}
W_{t}=(1-\alpha) \frac{Y_{t}}{L_{t}} \\
R_{t}=\alpha \frac{Y_{t}}{K_{t}}
\end{array}
$$

\subsection{Demanda Agregada e Exportações Líquidas}

O modelo também necessita de uma equação de demanda agregada:

$$
Y_{t}=C_{t}+I_{t}+N X
$$

As exportações líquidas são descritas como função da renda doméstica e da renda do resto do mundo:

$$
N X_{t}=\sigma Y_{t}+\varphi Y_{t}^{*}
$$

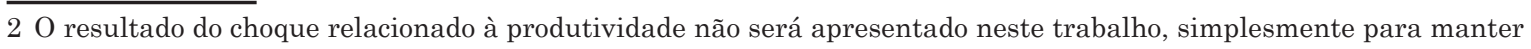
o foco renda mundial. 
onde $\sigma<0, \varphi>0$ são as sensibilidades das exportações líquidas em relação às rendas domésticas e do resto do mundo, respectivamente.

A renda do resto do mundo (renda mundial) segue um processo estocástico AR(1) da seguinte forma:

$$
Y_{t}^{*}=\rho^{*} Y_{t-1}^{*}+\varepsilon_{t}^{*}
$$

onde $\varepsilon_{t}^{*}$ é o termo de erro.

\subsection{Equilibrio}

Uma vez descrito o comportamento de cada agente do modelo. Nesta seção é apresentada a interação de todos os agentes para determinar o equilíbrio macroeconômico. Por conseguinte, o equilíbrio competitivo do modelo é alcançado por meio de um conjunto de dez equações: (2), (7), (8), (9), (10), (14), (15), (16), (17) e (18), que buscam representar o comportamento de dez variáveis endógenas $\left(Y, C, W, L, R, I, X, K, \mathrm{~A}\right.$ e $\left.Y^{*}\right)$ e duas variáveis exógenas $\left(\varepsilon, \varepsilon^{*}\right)$.

\section{Calibragem}

Este trabalho usou o procedimento de calibragem de duas maneiras para obter os parâmetros do modelo estrutural: o primeiro procedimento foi calibrar os dados $(\beta, \gamma, \delta, \alpha, \rho)$ do trabalho de Costa Junior et al (2012) (tabela 1); enquanto o segundo procedimento foi calibrar os parâmetros remanescentes $\left(\rho^{*}, \sigma, \varphi\right)$ (por meio de mínimos quadrados ordinários), usando os dados de crescimento das exportações líquidas, crescimento da renda doméstica e crescimento da renda mundial (figura 1$)^{3}$, conforme a regressão abaixo:

$$
\triangle N X=2,79+2,62 \triangle P I B_{E U A}-3,49 \triangle P I B
$$

$$
(0,5) \quad(1,8) \quad(-3,6)^{4}
$$

\section{Resultados}

Nesta seção, são examinadas as propriedades dinâmicas do modelo usando as funções impulso-resposta. tanto a formatação do modelo foi rodada na plataforma Dynare ${ }^{56}$

3 A renda dos Estados Unidos foi usada como proxy para a renda mundial.

4 Entre parênteses é apresentada a estatística t. Sendo que o resultado é que o PIB dos EUA é significante em $10 \%$ e o PIB brasileiro em 5\%. 
Tabela 1 - Distribuição a posteriori.

\begin{tabular}{cccc}
\hline $\begin{array}{c}\text { Parâme- } \\
\text { tro }\end{array}$ & Média & \multicolumn{2}{c}{$\begin{array}{c}\text { Intervalo de Con- } \\
\text { fiança }\end{array}$} \\
\hline$\beta$ & 0,9893 & 0,9864 & 0,9925 \\
$\gamma$ & 0,6996 & 0,6962 & 0,7030 \\
$\delta$ & 0,0507 & 0,0467 & 0,0551 \\
$\alpha$ & 0,3314 & 0,3011 & 0,3524 \\
$\rho$ & 0,9763 & 0,9394 & 0,9994 \\
\hline \multicolumn{2}{l}{ Fonte: Modificada de Costa Junior et al (2013). }
\end{tabular}

\subsection{Funções Impulso-resposta}

A figura 2 apresenta os resultados referente ao choque na renda mundial. Nota-se um efeito positivo no produto $(\mathrm{Y})$, na oferta de trabalho $(\mathrm{L})$ e no retorno do capital (R), enquanto um efeito negativo no consumo (C), no nível dos salários (W), no estoque de capital (K) e nos investimentos (I). O produto incialmente expande em $0,6 \%$ e gradativamente retorna ao seu estado estacionário em 15 períodos. Ocorre uma queda de aproximadamente $2 \%$ no consumo, tendo uma recuperação gradativa até voltar ao seu estado estacionário no período 40. As variáveis nível salarial $(\mathrm{W})$, trabalho $(\mathrm{L})$ e retorno do capital $(\mathrm{R})$ apresentam uma flutuação insignificante (abaixo de 1\%). Já os investimentos (I) caem aproximadamente $1 \%$, assim como no caso do produto, essa variável retorna ao seu estado estacionário em 15 períodos.

O resultado de duas outras variáveis merece uma maior atenção: o estoque de capital (K) cai em $1 \%$ e esse valor vai aumentando por 10 períodos, então ocorre uma inflexão na sua tendência; e as exportações líquidas aumentam em 3,5\% e retorna ao seu estado estacionário gradativamente até o período 40. Nota-se que o canal de propagação do choque na renda mundial $\left(\mathrm{Y}^{*}\right)$ é a exportação líquida (NX), essa variável, na medida em que recebe a influência do choque, aumenta de valor, o que eleva a renda doméstica, em seguida a renda doméstica aumenta o poder de compra das famílias, diminuindo o valor da exportação líquida (devido ao crescimento das importações), no período seguinte o efeito é menor do que o inicial, e esse mecanismo levará o modelo de volta ao seu estado estacionário.

\section{Conclusões}

O objetivo deste trabalho foi analisar o comportamento da economia brasileira em relação a um choque no nível de renda mundial, o qual aumenta as exportações líquidas brasileira, e essa variável é o canal de propagação desse choque. 
Percebe-se que o produto aumenta em relação ao choque na renda mundial, isso devido ao efeito positivo nas exportações líquidas, contudo esse crescimento é irrelevante, devido às quedas no consumo e no investimento. $\mathrm{O}$ rearranjamento dos fatores de produção é algo que chama a atenção, isso ocorre pelo crescimento da demanda por trabalho devido a queda no nível dos salários e aumento do retorno do capital. Entretanto, essas variáveis voltam ao seu estado estacionário dentro do período simulado.

Este trabalho pretendeu contribuir com um estudo das flutuações de curto prazo das exportações líquidas originadas por choques na renda mundial. Algumas conclusões ficam limitadas pela falta de um mercado financeiro e do Governo. Então, a sugestão para trabalhos futuros seria incorporar esses setores para uma análise mais completa.

Figura 2 - Funções impulso-resposta para o choque na renda mundial.
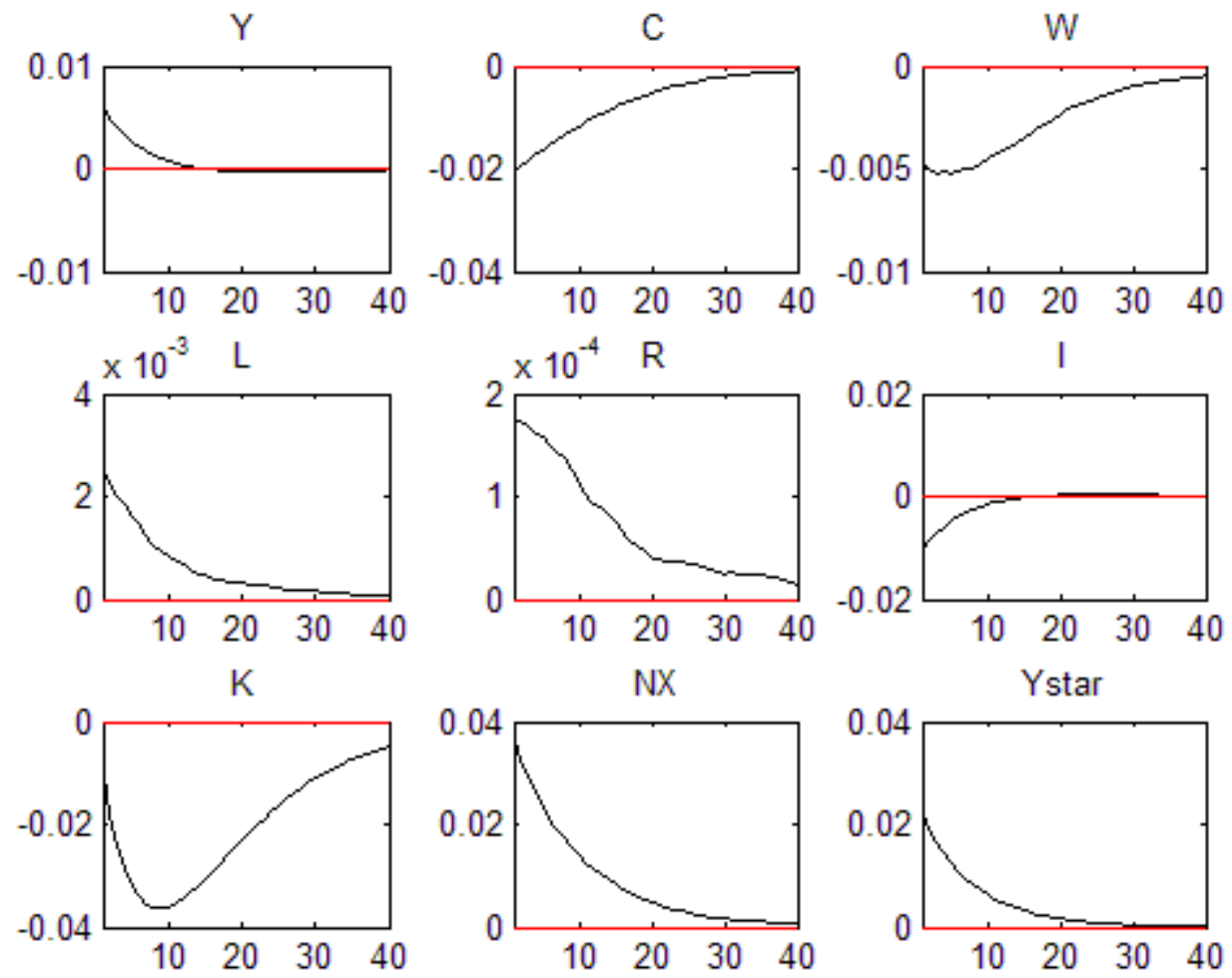

Fonte: Elaborada pelo autor.

\section{Referências}

Brock, W.; e Mirman, L. (1972). "Optimal economic growth and uncertainty: The discounted case." Journal of Economic Theory, 4:479-513.

Canova, F. (2007). Methods for Applied Macroeconomic Research. New Jersey: Princeton University Press.

Cass, D. (1965). "Optimum growth in an aggregative model of capital accumulation." Review of Economic Studies, 32:233-240. 
Costa Jr.; C. J.; Sampaio, A. V.; Gonçalves, F. O. (2012). "Transferência de Renda como Modelo de Crescimento Econômico.” Economia e Tecnologia, Vol. 8, n. 4, p. 17-32, 2012.

Ipeadata. Disponível em: <www.ipeadata.gov.br> Acesso em 10 de março de 2013.

Koopmans, T. (1965). "On the concept of optimal economic growth." The Econometric Approach to Development Planning, Amsterdam.

Kydland, F.; e Prescott, E. (1982). "Time to build and aggregate fluctuations." Econometrica, 50:1350-1372.

Ramsey, F. (1927). "A contribution to the theory of taxation." Economic Journal, 37(145):47-61.

Ramsey, F. (1928). "A mathematical theory of saving." Economic Journal, 38(152):543-559. 
